\title{
Ultrafast NEXAFS spectroscopy in the lab using laser-based sources and advanced X-ray optics
}

Holger Stiel, Julia Braenzel, Johannes Tuemmler, Adrian Jonas, Ioanna Mantouvalou, et al.

Holger Stiel, Julia Braenzel, Johannes Tuemmler, Adrian Jonas, Ioanna Mantouvalou, Alexei Erko, Anke Heilmann, Martin Moerbeck-Bock, Matthias Schnuerer, "Ultrafast NEXAFS spectroscopy in the lab using laser-based sources and advanced X-ray optics," Proc. SPIE 11886, International Conference on X-Ray Lasers 2020, 1188612 (8 July 2021); doi: $10.1117 / 12.2593295$ 


\title{
Ultrafast NEXAFS spectroscopy in the lab using laser-based sources and advanced $\mathrm{X}$-ray optics
}

\author{
Holger Stiel $^{\text {a,b }}$, Julia Braenzel ${ }^{\mathrm{a}}$, Johannes Tuemmler ${ }^{\mathrm{a}, \mathrm{b}}$, Adrian Jonas ${ }^{\mathrm{b}, \mathrm{c}}$, Ioanna Mantouvalou ${ }^{\mathrm{b}, \mathrm{c}, \mathrm{d}}$, \\ Alexei Erko $^{\mathrm{e}}$, Anke Heilmann ${ }^{\mathrm{a}}$, Martin Moehrbeck-Bock ${ }^{\mathrm{a}}$, Matthias Schnuerer ${ }^{\mathrm{a}}$ \\ ${ }^{a}$ Max-Born-Institute, Max-Born-Strasse 2a, 12489 Berlin Germany \\ ${ }^{\mathrm{b}}$ Berlin Laboratory for innovative X-ray technologies, 10623 Berlin, Germany \\ ${ }^{c}$ Institute for Optics and Atomic Physics, Technical University of Berlin, Hardenbergstr. 36, 10623 \\ Berlin, Germany \\ ${ }^{\mathrm{d}}$ Helmholtz Zentrum Berlin, Albert-Einstein-Str. 15, 12489 Berlin, Germany \\ ${ }^{\mathrm{e}}$ Institut für angewandte Photonik eV., Rudower Chaussee 20-21, 12489 Berlin, Germany
}

\begin{abstract}
Laboratory based laser driven short pulse X-ray sources like laser produced plasmas (LPP) and high harmonic generation (HHG) exhibit a great potential for spectroscopy in the soft X-ray range. These sources are complementary to large scale facilities like synchrotrons or free electron lasers. For applications of LPP or HHG sources for time-resolved X-ray absorption spectroscopy in the water window or beyond a high photon flux is crucial. The available photon flux strongly depends on energy, pulse duration and repetition rate of the pump laser. Depending on the experimental needs in timeresolved experiments pulse durations of the X-ray pulse ranging from nanoseconds to sub-femtoseconds are required.

In our contribution we will present a highly brilliant LPP source emitting soft X-rays in the photon energy range between 50 and $1500 \mathrm{eV}$ based on CPA and thin disk laser technology as well as the high average power thin disk laser based OPCPA system for high photon flux HHG.

In addition we present a new generation of reflection zone plates on spherical substrates, that promises a remarkable high resolution over a wide spectral range making it an ideal and highly efficient diffractive optic for time-resolved NEXAFS experiments in the lab.
\end{abstract}

Keywords: Laser produced plasma, HHG, pump-probe, reflection zone plates, water window, L-edges, X-ray absorption, NEXAFS

\section{INTRODUCTION}

Laboratory based laser driven short pulse X-ray sources like laser produced plasmas (LPP) and high harmonic generation (HHG) exhibit a great potential for spectroscopy in the soft X-ray range [1-4]. These sources are complementary to large scale facilities like synchrotrons or free electron lasers. For applications of LPP or HHG sources for time-resolved X-ray absorption spectroscopy in the water window $(280-540 \mathrm{eV})$ or beyond $(550-1500 \mathrm{eV}$, e.g. at the transition metal Ledges or M-edges of rare earth metals) the available photon flux is crucial [5]. The available photon flux strongly depends on energy, pulse duration and repetition rate of the pump laser. Depending on the experimental needs in timeresolved experiments pulse durations of the X-ray pulse ranging from nanoseconds to sub-femtoseconds are required maintaining a jitter-free synchronization with the optical pump pulse. Due to the source emission characteristics (pulse 
duration, radiation geometry and coherence) LPP and HHG offer also complementary research possibilities for various dynamical processes at different timescale

Due to physical limitations, the total X-ray photon number per pulse of LPP and HHG sources is relatively low. Therefore, a very efficient optical system for spectroscopy and monochromatization is needed. The existing soft X-ray spectrometers on the basis of replicas of spherical, reflective X-ray gratings have low efficiency, in the order of one percent. Therefore their application in time-resolved (pump-probe) X-ray absorption experiments is very limited.

In this contribution, we characterize two laser based soft X-ray sources and discuss their application in near edge X-ray absorption fine structure (NEXAFS) experiments using two different spectrometer setups. We will discuss the properties of a new X-ray optical system based on reflection zone plate arrays on bent substrates with an efficiency which is one order of magnitude higher than that of existing spectrometers.

\section{EXPERIMENTAL}

\section{Laser produced plasma source (LPP)}

The LPP soft X-ray source is pumped by a homemade CPA thin disc laser (TDL) system. The TDL system consists of a front-end (YGW oscillator and regenerative amplifier), a stretcher and a Yb:YAG thin disc regenerative amplifier (cp. Fig. 1) The output of the regenerative amplifier is compressed using a grating compressor to a pulse duration of $1.6 \mathrm{ps}$. The TDL operates at $1030 \mathrm{~nm}$ with $100 \mathrm{~Hz}$ repetition rate and delivers pulses with an energy of $120 \mathrm{~mJ}$ after compression. In our experimental setup the laser is focused with a f5 optic at an angle of $\sim 45^{\circ}$ on a metallic rotating target cylinder. In the focal region with a FWHM of $17 \mu \mathrm{m}$ we reach $60 \%$ energy content corresponding to an intensity of about $2 \times 10^{16} \mathrm{~W} / \mathrm{cm}^{2}$ on the target. The plasma created by the laser pulse emits a wide soft X-ray spectrum. We measured the soft X-ray source size with a pinhole camera setup to be at least 2x the laser focal size (FWHM) from which the incoherent soft X-ray radiation (SXR) is uniformly emitted. For debris protection a thin glass plate after the entrance window of the vacuum chamber and an optional $900 \mathrm{~nm}$ Mylar foil after the target on the entrance of the soft $\mathrm{X}$-ray beamline were used. Fig. 1 shows the scheme of the laser and beam guiding system in our setup.

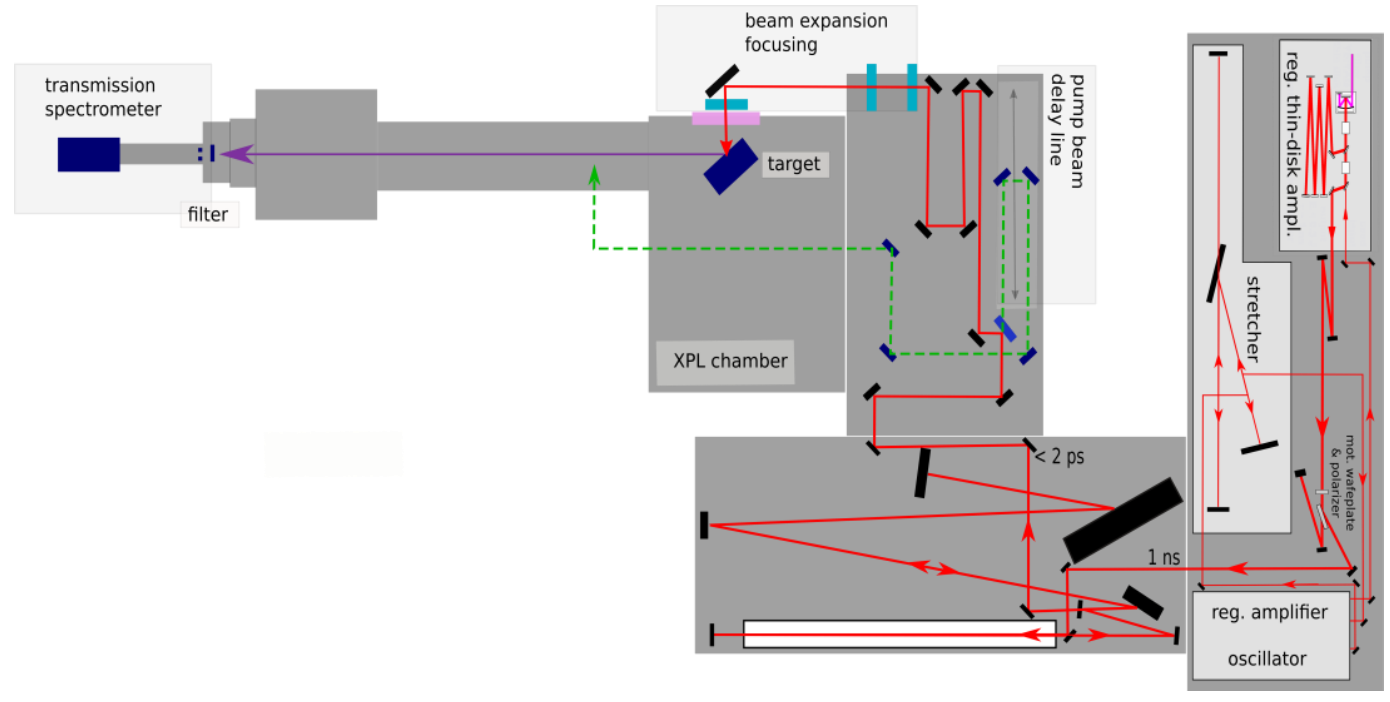

Figure 1. Schematic of the LPP source setup including the CPA thin disk pump laser

\section{High Harmonics Generation (HHG) source}

The HHG soft X-ray source is driven by a state-of-the-art high power OPCPA system [2]. A schematic of the setup is shown in Fig. 2. The high average power OPCPA system is based on a single Yb:YAG thin disk laser (Dira 500-10, Trumpf Scientific Lasers) delivering $500 \mathrm{~W}$ average power at a pulse repetition rate of $10 \mathrm{kHz}$. This output is used both as pump and signal generation source for the OPCPA system. For this purpose, it is split into two arms. The low power arm contains $0.5 \mathrm{~mJ}$ pulses, compressed to below one picosecond, which are delivered to a front-end manufactured by Fastlite. The high power arm contains the remaining power, compressed to a pulse duration of about 2 ps. In the frontend, the seed is obtained by super continuum generation and subsequent difference frequency generation. Two PPLNbased OPA stages are used as pre-amplifiers, and an acousto-optic programmable dispersive filter serves as pulse-shaper. 
The output pulses of the front-end have an energy of about $2.7 \mu \mathrm{J}$ and extend from $1800 \mathrm{~nm}$ to $2600 \mathrm{~nm}$, with a central wavelength of $2.1 \mu \mathrm{m}$. In the subsequent power amplification, the pulse energy is boosted to more than $3 \mathrm{~mJ}$ using two home-built OPCPA stages. The first stage is pumped with $130 \mathrm{~W}$ average power and amplifies the $2.1 \mu \mathrm{m}$ pulses to about $1 \mathrm{~mJ}$ using $\mathrm{BiBO}$ as nonlinear crystal. In the second stage, a YCOB crystal pumped with $320 \mathrm{~W}$ is used to increase the pulse energy to its final value. A part of this power is separated to be used as pump beam in pump-probe experiments, so that $2.8 \mathrm{~mJ}$ are available for HHG. The pulses are compressed using a $41 \mathrm{~mm}$ thick suprasil glass block, placed under an angle in the beam such that it compensates the dispersion accumulated in the setup. The compressed pulse duration typically is about $26 \mathrm{fs}$, with a spectral width of more than $400 \mathrm{~nm}$ FWHM.

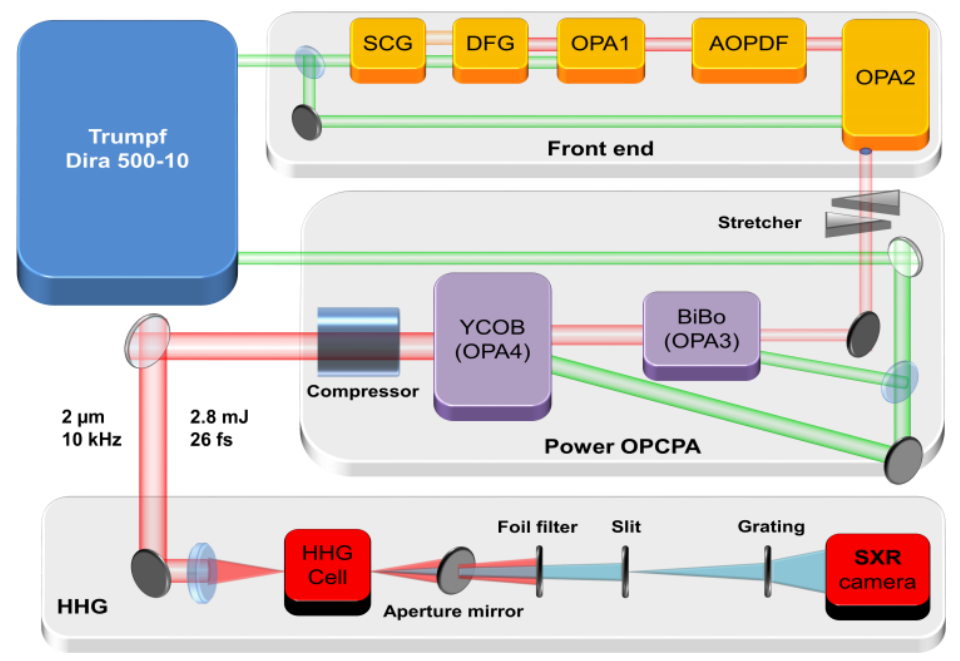

Figure 2. Schematic of the OPCPA and HHG setup.

The compressor serves at the same time as entrance window in the vacuum setup used for HHG. The beam is focused by a $500 \mathrm{~mm}$ lens into a differentially pumped $5 \mathrm{~mm}$ long gas cell filled with up to $1.5 \mathrm{bar}$ of helium. Behind the cell, most of the transmitted IR radiation is reflected by a mirror, where the SXR radiation can pass through a hole in its center. The remaining IR light is blocked by one or more thin foil filters, so that only the X-rays pass through a slit and a transmission grating onto an X-ray CCD camera.

\section{Photon Flux Evaluation}

Both soft X-ray sources were characterized using a transmission grating spectrometer (TGS) setup schematically shown in Fig. 3). It consists of a $10.000 \mathrm{l} / \mathrm{mm}$ free standing $\mathrm{Si}_{3} \mathrm{~N}_{4}$ transmission grating [6] and an IDus $420 \mathrm{CCD}$ detector (ANDOR, 1024×255 pixels). Thin metal foils ( $\mathrm{Al}, \mathrm{Zr}$ ) were used to shield the IR light. The foils also provide absorption edges for the spectral calibration. The detector was mounted on a rail that allowing to change the detection angle in the range of \pm 10 degree. Our setup provided a detection angle of $2.1 \times 10^{-8} \mathrm{sr}$ with a slit $3 \mathrm{~mm}$ in height and $50 \mu \mathrm{m}$ in width, that was placed just before the transmission grating. The setup was calibrated at the German national metrology institute, Physikalisch-Technische Bundesanstalt (PTB), and can therefore be used to account for the absolute number of photons generated in the LPP and HHG process.

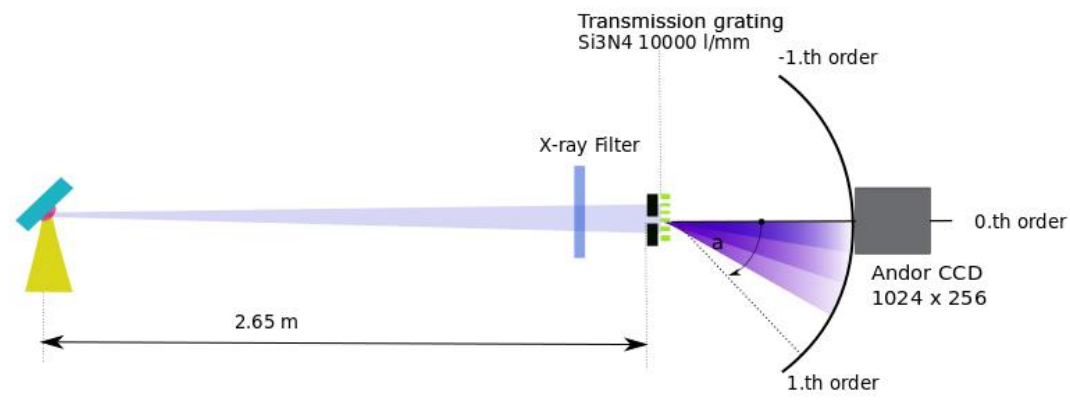

Figure 3. Calibrated transmission grating spectrometer (TGS) used for the evaluation of the photon flux of the LPP. For the evaluation of the photon flux of the HHG a similar setup (not shown) has been used. 


\section{Reflection zone plate spectrometer}

For our NEXAFS investigations with the LPP shown below we used a spectrometer setup with reflection zone plates (RZP) on planar substrates. It is described in more detail in [5].

In order to further improve spectrometer efficiency, spectral resolution and energy range in NEXAFS investigations we rely on a reflection zone plate array (RZPA) on a spherical substrate. The main references concerning a possible optical layout solution is the RZPA spectrometer as published in [7,8]. Recent advancements with RZPAs on a spherical substrate are reported in [9]. As an option, the RZPA provides up to four different RZPs, each with the same resolving power, together covering the energy range $(150-700) \mathrm{eV}$ with HHG source and $(210-1400) \mathrm{eV}$ with LPP source. The spectrometers provide a single-shot online monitoring of the initial spectra of the source, energy resolving power $\mathrm{E} / \Delta \mathrm{E}$ above 1000 and peak efficiency up to $25 \%$. As a detector typical X-ray CCD or sCMOS camera with pixel size of 13.5 $\mu \mathrm{m} \times 13.5 \mu \mathrm{m}$ can be used. We have projected two related spectrometer designs for the LPP and HHG source respectively. As an example Fig. 4 shows the optical layout of the proposed LPP spectrometer.

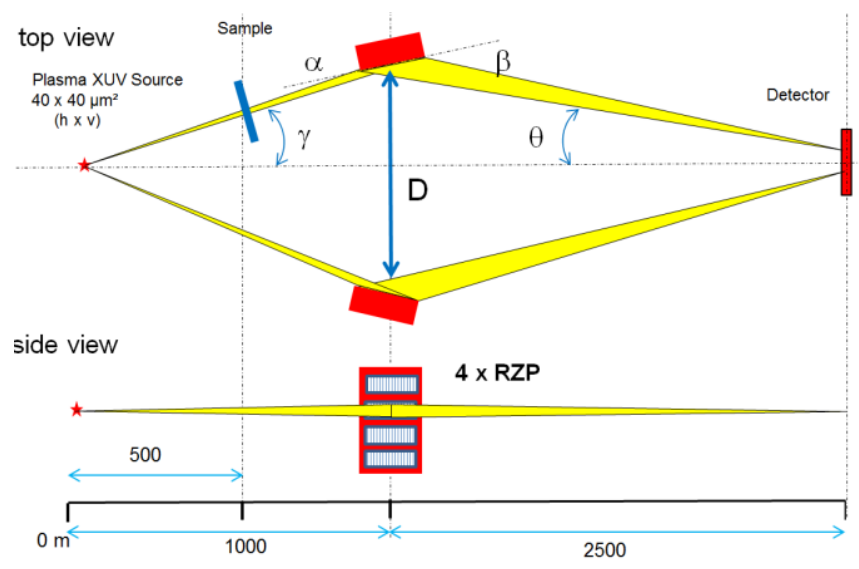

Figure 4. Optical layout of the LPP spectrometry system. See Table 1 for the notation and data.

The X-ray beam from the LPP source is transmitted through a sample, which is located in a distance of $0.5 \mathrm{~m}$ from the target. The parameters of RZPAs in reference and signal channels are identical. The position of the CCD detector is assumed to be fixed. Therefore, in order to cover the full energy region of interest four RZPs are designed on the same substrate. Parameters of these RZP structures are shown in table 2. Each RZP has an aperture of $6 \mathrm{~mm}$ sagittal and $80 \mathrm{~mm}$ meridional fabricated on a spherical substrate with a radius of $45 \mathrm{~m}$.

Table 1. Optical layout parameters.

\begin{tabular}{|c|c|}
\hline Input angle $\gamma$ & $4.34^{\circ}$ \\
\hline Output angle $\theta$ & $1.31^{\circ}$ \\
\hline RZPA separation distance D (mm) & 151.8 \\
\hline
\end{tabular}

Table 2. Parameters of the optical elements within RZPA (signal and reference channel)

\begin{tabular}{|c|c|c|c|c|}
\hline Parameter & RZP 1 & RZP 2 & RZP 3 & RZP 4 \\
\hline Designed energy (eV) & 265 & 425 & 680 & 1070 \\
\hline $\begin{array}{c}\text { Energy range (eV) } \\
\text { on CCD, aperture: } 27.6 \mathrm{~mm}\end{array}$ & $210-345$ & $340-555$ & $550-880$ & $850-1400$ \\
\hline Meridional line density (1/mm) & $298-303-308$ & $478-487-494$ & $765-778-791$ & $1203-1225-1245$ \\
\hline Grazing input/diffraction angles & \multicolumn{4}{|c|}{$\alpha=2.5^{\circ}, \beta=3.65^{\circ}$} \\
\hline
\end{tabular}

The results of the calculation of expected efficiency and energy resolving power are shown in figure $5 \mathrm{a}$ and $5 \mathrm{~b}$ accordingly. 


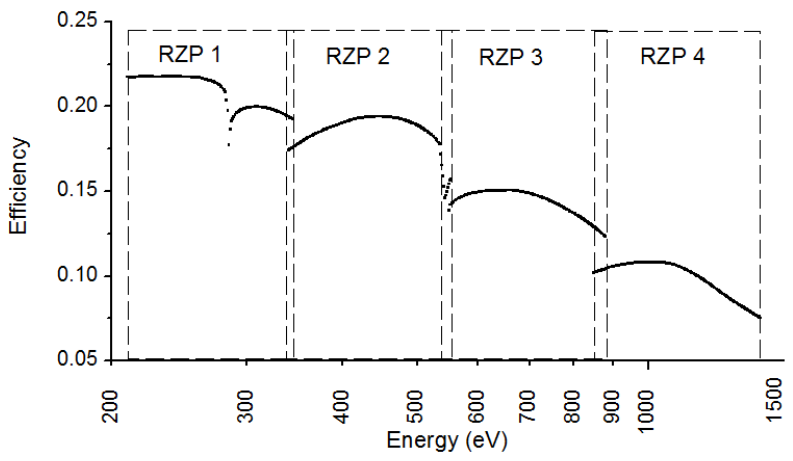

Figure 5a. Calculated RZP efficiencies, assuming Au coating with a CO contamination layer $(2 \mathrm{~nm})$ on the top of Au.

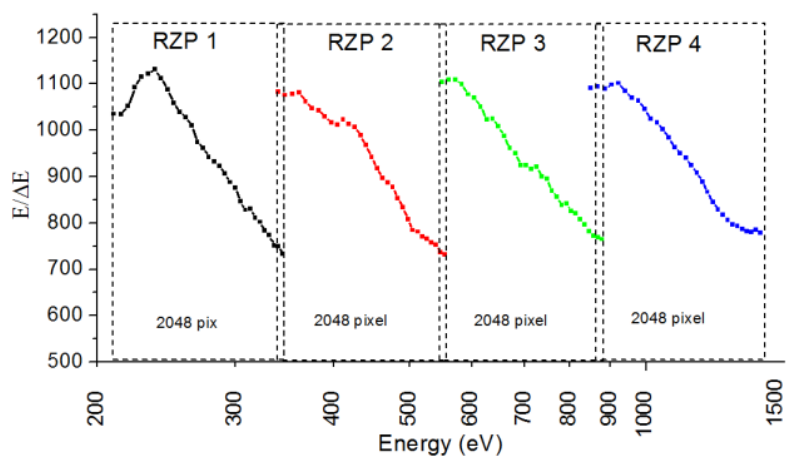

Figure 5b. Energy resolving power of RZPs $1-4$, covering the energy range $(210-1400) \mathrm{eV}$.

\section{RESULTS}

\section{Photon flux and spectra of the LPP source}

Using the calibrated TGS setup (cf. Fig. 3) soft X-ray spectra were measured with different target materials copper and tungsten. For the evaluation we averaged datasets of at least $10 \times 10 \mathrm{~s}$ at the laser's repetition rate of $100 \mathrm{~Hz}$. Fig. 6 shows as an example the measured spectra emitted by a tungsten target and filtered by $\mathrm{Zr}$ (298nm thickness) or $\mathrm{Al}$ (200nm) foils. Characteristic absorption edges by either filter $(\mathrm{Al}, \mathrm{Zr})$, filter contaminations $(\mathrm{O}, \mathrm{C})$ and the supporting structure of the transmission grating $\left(\mathrm{Si}_{3} \mathrm{~N}_{4}\right)$ can be identified in the tungsten spectra. The photon flux of the LPP source was evaluated from the averaged raw data (counts/s) with the efficiency of the transmission grating (theoretical efficiency function normalized to a fixed calibrated photon energy), filter transmission characteristics (calculations using Henke database [10]) and the calibrated CCD detector. Fig. 7a presents the resulting flux in photons $/ \mathrm{s} * \mathrm{sr}$ in $0.1 \%$ bandwidth for the tungsten target and Fig. 7b for selected Cu-emission lines. Both figures show, that the LPP source emits in a wide spectral range $(50-1500 \mathrm{eV})$ at least $10^{11}$ photons/s *sr @0.1\% bandwidth.
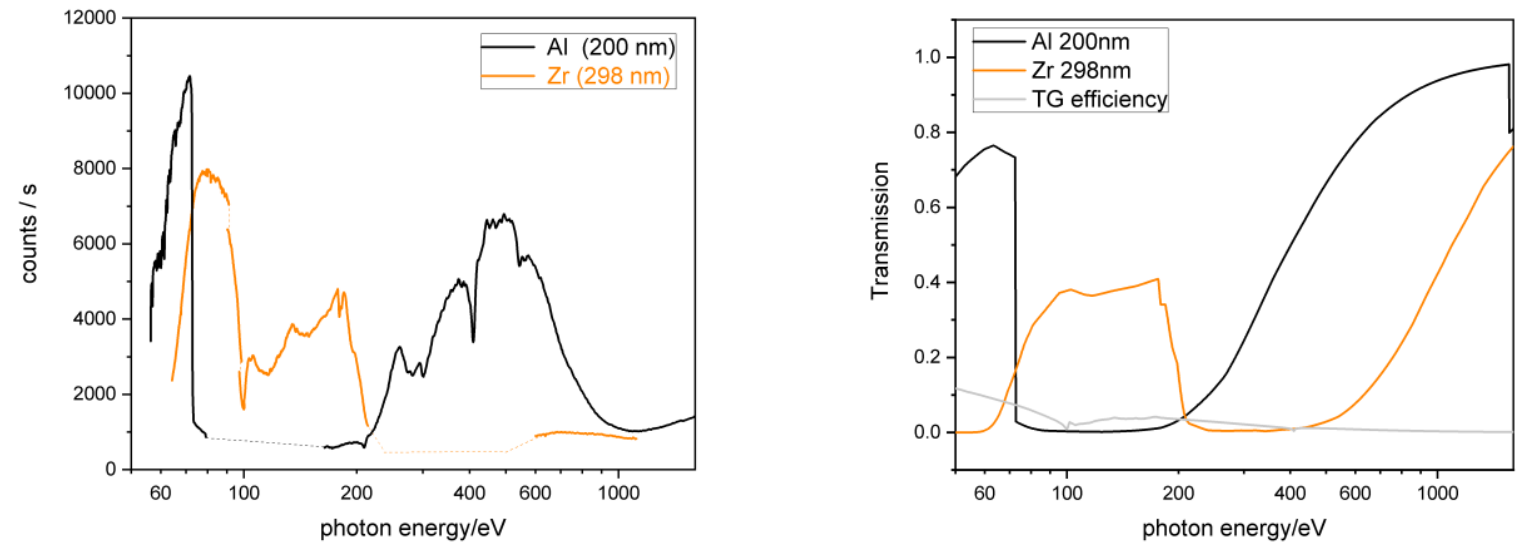

Figure 6. Measured emission spectra of the LPP source (left) filtered by $\mathrm{Zr}$ and Al foils (right) Target: tungsten. 

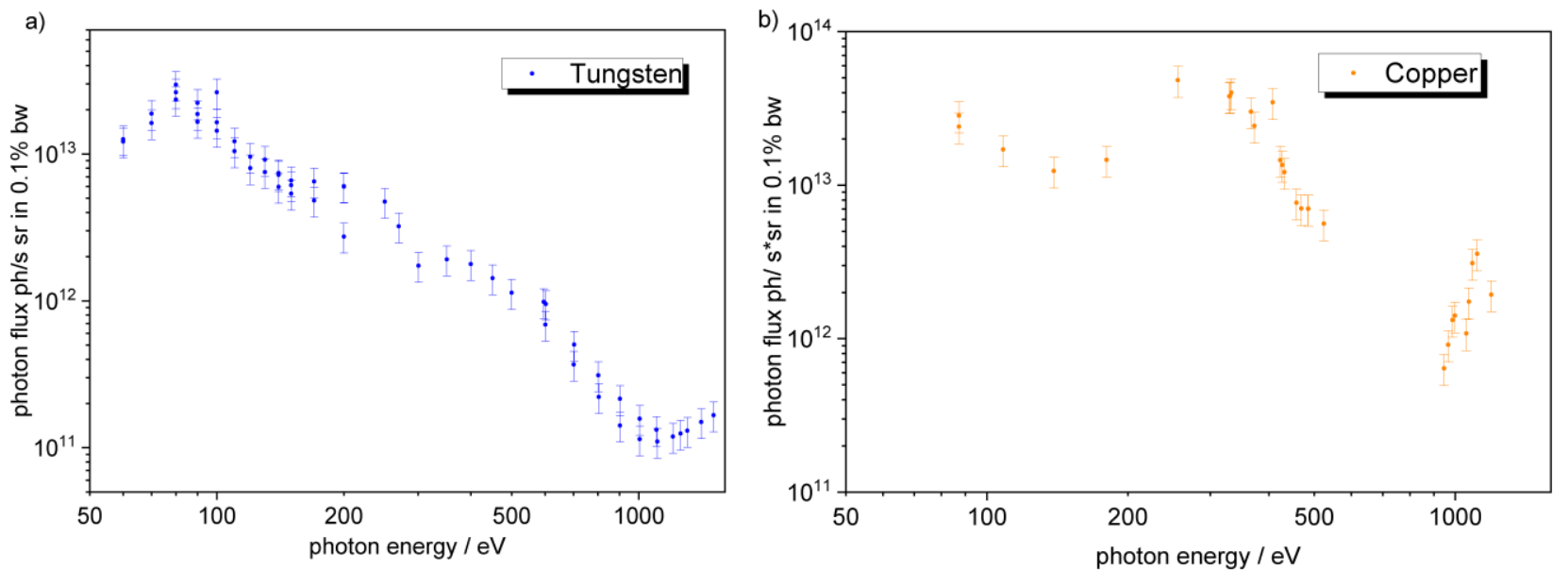

Figure 7. Photon flux of the LPP source corrected for filter transmission, grating efficiency and detector response for the tungsten (a) and the copper target (b).

\section{Photon flux of HHG}

Two typical spectra recorded with our HHG source can be seen in Fig. 8. The green curve was taken with only an aluminum foil filter, whereas for the purple curve, an additional titanium foil was inserted. As can be seen from Fig. 8 the Ti L-edge is clearly visible. Using the calibrated spectrometer setup described above, the foil filter transmission curves from [10] and an estimation of the ratio of photons passing through the spectrometer entrance slit, we calculated a photon flux of up to $10^{6}$ photons $/ \mathrm{eV} / \mathrm{s}$ at a photon energy of $450 \mathrm{eV}$. The obtained flux is comparable to already reported values obtained with laser drivers of lower repetition rate [11-13] but the achieved single pulse conversion from IR to SXR energy is lower and needs further investigation. One has to admit that, in contrast to our measurements, the majority of reported values is not based on calibrated measurement devices.

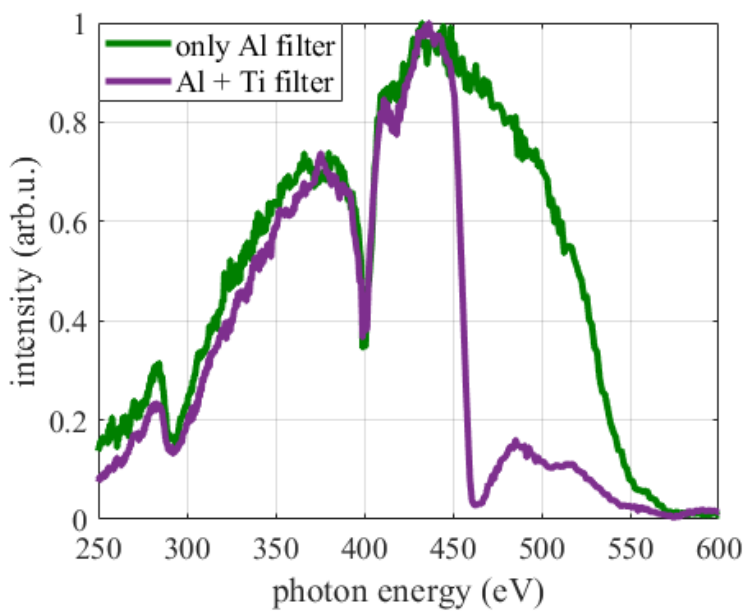

Figure 8. Typical HHG spectrum obtained with the transmission grating spectrometer. Gas medium: He at 1.8 bar. Laser parameters: $9 \times 10^{14} \mathrm{~W} / \mathrm{cm}^{2}, 25 \mathrm{fs}, 10 \mathrm{kHz}$. Filters: Al $-200 \mathrm{~nm}, \mathrm{Ti}-200 \mathrm{~nm}$.

\section{X-ray absorption (XAS) investigations}

We used the TGS spectrometer (cp. Fig. 3) and a spectrometer setup based on plane RZP substrates [5] to demonstrate the capability of both sources for NEXAFS investigations on thin metal films. Fig. 9 shows NEXAFS a spectrum of a Ti 
foil $(200 \mathrm{~nm})$ registered with the TGS and the HHG source in comparison with the spectrum of the same sample registered using the LPP source and a spectrometer with plane RZP substrates. As can be seen from Fig. 9 both the resolution (cp. edge jumps of both spectra) and the signal to noise ratio of the spectrum could be significantly increased in the case of the RZP spectrometer. Nevertheless in both spectra characteristics features of the Ti L-edge absorption are visible. The broad spectrum of the LPP source together with the highly efficient RZP spectrometer provides the possibility to investigate also extended X-ray absorption fine structures (EXAFS) at L-edges of transition metals. As an example Fig. 10 shows the XAS spectrum of a Ni foil covering a large photon energy range. EXAFS oscillations are clearly visible.

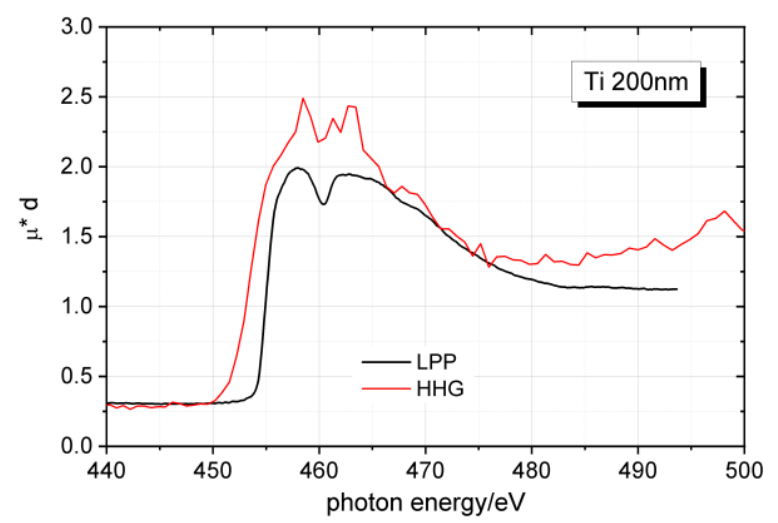

Figure 9. NEXAFS spectra of a Titanium foil $(200 \mathrm{~nm})$ measured with a HHG source and a TGS spectrometer in comparison with a spectrum recorded using the LPP source and a spectrometer based on plane RZP substrates.

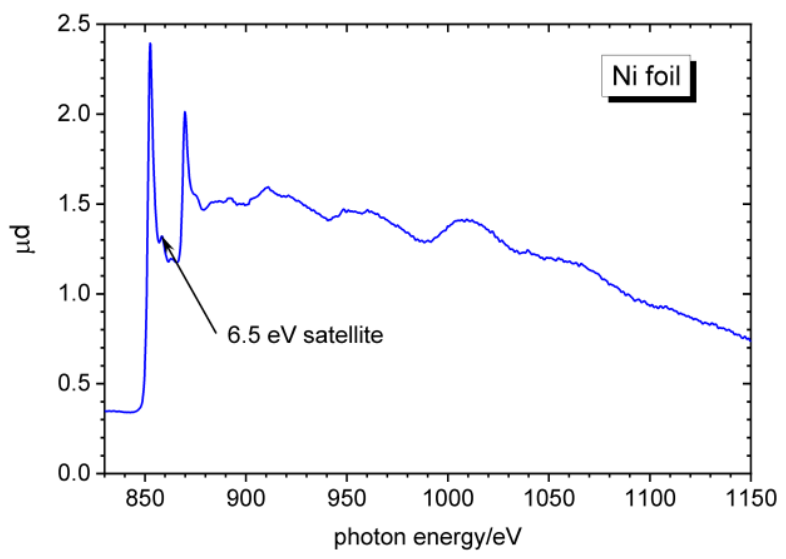

Figure 10. L-edge XAS spectrum of a Ni foil $(200 \mathrm{~nm})$ showing both near edge structures as well as oscillations in the EXAFS region.

The spectrum was recorded with a RZP spectrometer based on plane substrates.

\section{CONCLUSIONS AND FUTURE}

We have developed two highly brilliant soft X-ray sources for time-resolved X-ray absorption spectroscopy in the lab. Both sources rely on thin disc laser technology for the pump laser system. The HHG source delivers fs soft X-ray pulses in a photon energy range covering the whole water window region whereas the LPP source covers a larger photon energy range with pulse durations in the $10 \mathrm{ps}$ range.

We have shown that due to their high efficiency and high resolving power reflection zone plates are very promising dispersive elements for NEXAFS experiments in the lab. Reflection zone plate arrays on bent substrates exhibit a higher efficiency maintaining a high spectral resolution over a broader photon energy range. Therefore these optical elements are very well suited for photon-hungry time-resolved NEXAFS experiments. The minimum detectable change in optical density ( $\triangle \mathrm{OD}$ ) induced by the pump pulse in these experiments depends on the flux on the detector (photon statistics) as 
well as on data acquisition rate. We expect that the combination of highly efficient RZPA described in this paper with advanced sCMOS detectors [14] will allow in future the detection of $\triangle \mathrm{OD}<10^{-3}$ even with sources delivering only a moderate photon flux per pulse.

For the existing HHG source, future work will concentrate on the increase of photon flux and cutoff energy by finetuning of the laser parameters and the gas cell design. Moreover, a second OPCPA system with a similar design, but a central wavelength of $3 \mu \mathrm{m}$ is currently under construction, which will allow to increase the HHG cutoff beyond $600 \mathrm{eV}$. Hence, it will be possible to perform cutting-edge experiments using laboratory-scale coherent and incoherent SXR sources.

Both sources enable us to perform NEXAFS - studies in a complementary way. It is important to develop laser driver, source-technology, SXR-optics and detection methods in context to meet and to exploit the specifics of laboratory scale laser based systems.

\section{ACKNOWLEDGEMENT}

This work has been supported by EFRE project "Nanomovie" 1.8/10 and ProFit project "MOSFER" \#10168892.

\section{REFERENCES}

[1] I. Mantouvalou, K. Witte, D. Grötzsch, M. Neitzel, S. Günther, J. Baumann, R. Jung, H. Stiel, B. Kanngießer, and W. Sandner, "High average power, highly brilliant laser-produced plasma source for soft X-ray spectroscopy," Review of Scientific Instruments, 86(3), 035116 (2015).

[2] T. Feng, A. Heilmann, M. Bock, L. Ehrentraut, T. Witting, H. Yu, H. Stiel, S. Eisebitt, and M. Schnürer, "27 W $2.1 \mu \mathrm{m}$ OPCPA system for coherent soft X-ray generation operating at $10 \mathrm{kHz}$," Optics Express, 28(6), 8724$8733(2020)$.

[3] B. Buades, D. Moonshiram, T. P. H. Sidiropoulos, I. León, P. Schmidt, I. Pi, N. Di Palo, S. L. Cousin, A. Picón, F. Koppens, and J. Biegert, "Dispersive soft X-ray absorption fine-structure spectroscopy in graphite with an attosecond pulse," Optica, 5(5), 502-506 (2018).

[4] P. Wachulak, M. Duda, A. Bartnik, A. Sarzynski, L. Wegrzynski, M. Nowak, A. Jancarek, and H. Fiedorowicz, "Compact system for near edge X-ray fine structure (NEXAFS) spectroscopy using a laser-plasma light source," Optics Express, 26(7), 8260-8274 (2018).

[5] A. Jonas, H. Stiel, L. Glöggler, D. Dahm, K. Dammer, B. Kanngießer, and I. Mantouvalou, "Towards Poisson noise limited optical pump soft X-ray probe NEXAFS spectroscopy using a laser-produced plasma source," Optics Express, 27(25), 36524-36537 (2019).

[6] T. Wilhein, S. Rehbein, D. Hambach, M. Berglund, L. Rymell, and H. M. Hertz, "A slit grating spectrograph for quantitative soft x-ray spectroscopy,” Rev. of Sci. Instrum., 70(3), 1694-1699 (1999).

[7] A. Erko, C. Braig, J. Probst, and H. Löchel, "Novel x-ray optical systems for ultrafast spectroscopy and monochromators," SPIE Optical Engineering + Applications, 11110, (2019).

[8] A. Erko, C. Braig, and H. Löchel, "Spectrometers and monochromators for femtosecond soft x-ray sources," SPIE Optical Engineering + Applications, 11108, (2019).

[9] J. Probst, C. Braig, and A. Erko, "Flat Field Soft X-ray Spectrometry with Reflection Zone Plates on a Curved Substrate," Applied Sciences, 10(20), 7210 (2020).

[10] B. L. Henke, [X-Ray Attenuation Length], https://henke.lbl.gov/optical constants/atten2.html(2020).

[11] M. C. Chen, P. Arpin, T. Popmintchev, M. Gerrity, B. Zhang, M. Seaberg, D. Popmintchev, M. M. Murnane, and H. C. Kapteyn, "Bright, Coherent, Ultrafast Soft X-Ray Harmonics Spanning the Water Window from a Tabletop Light Source," Physical Review Letters, 105(17), 173901 (2010).

[12] V. Cardin, B. E. Schimdt, N. Thiré, S. Beaulieu, V. Wanie, M. Negro, C. Vozzi, V. Tosa, and F. Légaré, "Selfchannelled high harmonic generation of water window soft x-rays," Journal of Physics B: Atomic, Molecular and Optical Physics, 51(17), 174004 (2018).

[13] A. S. Johnson, D. R. Austin, D. A. Wood, C. Brahms, A. Gregory, K. B. Holzner, S. Jarosch, E. W. Larsen, S. Parker, C. S. Strüber, P. Ye, J. W. G. Tisch, and J. P. Marangos, "High-flux soft x-ray harmonic generation from ionization-shaped few-cycle laser pulses," Science Advances, 4(5), eaar3761 (2018).

[14] K. Desjardins, H. Popescu, P. Mercère, C. Menneglier, R. Gaudemer, K. Thånell, and N. Jaouen, "Characterization of a back-illuminated CMOS camera for soft x-ray coherent scattering," AIP Conference Proceedings, 2054(1), 060066 (2019). 